\title{
Nueva Generación de Heurísticas para Redes de Fibra Óptica WDM (Wavelength Division Multiplexing) bajo Tráfico Dinamico
}

\author{
Arturo B. Rodriguez ${ }^{(1)}$, Leonardo J. Ramirez ${ }^{(2)}$ y Jorge Chahuan ${ }^{(3)}$ \\ (1) Universidad Santiago de Chile, Departamento de Tecnología Industrial, Grupo de Investigación en \\ Nuevas Tecnologías (GINT), Santiago-Chile. (e-mail: arturo.rodriguez@usach.cl) \\ (2) Universidad Militar de Nueva Granada, División de Desarrollo Tecnológico e Innovación de la \\ Vicerrectoría de Investigaciones, Grupo de Investigación en Telemedicina (TIGUM), Bogotá, Colombia. \\ (e-mail: leonardo.ramirez@unimilitar.edu.co) \\ (3) Universidad de las Américas, Santiago-Chile. (email: jchahuan@udla.cl)
}

Recibido Feb. 16, 2015; Aceptado Abr. 20, 2015; Versión final Abr. 26, 2015, Publicado Oct. 2015

\begin{abstract}
Resumen
El presente artículo muestra una nueva heurística denominada Snake y sus posibilidades futuras para mejorar los indicadores de probabilidad de bloqueo y utilización de la red de transporte de datos. Para ello, se realizó la comparación de los algoritmos Recocido Simulado (Simulated Annealing), Algoritmos Genéticos, Búsqueda Tabú y el nuevo Algoritmo fueron simulados en la red óptica WDM NSFNET. Los resultados muestran un nuevo algoritmo que mejora la probabilidad de bloqueo pero no el indicador de utilización de la red. Los resultados muestran que hay un alto consumo de los recursos de red, lo que actúa negativamente para atender la demanda futura. Esto implica que hacen falta modificaciones para la mejora del proceso y que se deben desarrollar cambios en el algoritmo que permitan una mejora de ambos indicadores.
\end{abstract}

\section{New Generation of Heuristics for Wavelength Division Multiplexing (WDM) Optical Fiber Networks under Dynamic Traffic}

\begin{abstract}
This paper presents a new heuristic called Snake and future possibilities for improving the indicators of blocking probability and data transport networks utilization. For this, the algorithms of Simulated Annealing, Genetic Algorithms, Tabu Search Algorithm and the proposed Snake are simulated in the WDM optical network NSFNET. The results show the new algorithm improves the blocking probability but not the display network utilization. The results show that there is a high consumption of network resources which negatively acts to meet future demand. This implies that changes are needed to improve the process and that it is also necessary to develop the algorithms that allow an improvement in both indicators.
\end{abstract}

Keywords: simulated annealing; genetics algorithm; heuristic; WDM; Snake algorithm 


\section{INTRODUCCIÓN}

Los servicios que involucran transmisión de información, tales como: Aplicaciones web, video conferencia, email, transferencia de archivos, servicios on demand entre otros, y su incesante aumento han sometido a las redes WDM (Wavelength Division Multiplexing) a un tráfico cada vez más complejo. Un tráfico que por lo general fue estático, hoy es completamente dinámico. En las redes WDM se debe buscar una ruta de servicio y asignar una longitud de onda a dicha ruta, en escenarios de tráfico estático o dinámico, para el caso estático es optimizable con algoritmos convencionales muy revisados en la literatura (Assis et al.,2010; Rodríguez et al., 2010; Charbonneau et al., 2012), esto es debido a un universo de soluciones invariante por largos periodos de tiempo, que permite la optimización para establecer una conexión, es decir encontrar la mejor ruta y longitud de onda; para el caso dinámico el universo de soluciones es variante lo que imposibilita su optimización, por lo tanto los algoritmos heurísticos son especialmente importante para la solución de este problema. Debido a que el tráfico en estas redes es definitivamente dinámico, las investigaciones actuales están orientadas al desarrollo de heurísticas que permitan mejorar dos indicadores de relevante importancia para las redes ópticas. El primer indicador es la probabilidad de bloqueo, que determina las posibilidades de servicio de una solicitud entrante, el segundo indicador es la utilización de la red, que determina la disponibilidad de establecimiento de la conexión para las solicitudes futuras. Los algoritmos heurísticos deben ser probados en condición de mejorar estos indicadores (Rodríguez et al., 2010).

Una ruta y su longitud de onda asociada es denominada LightPath (LP) y el problema de determinar el LP es denominado RWA (Routing Wavelength Assignment). La solución del RWA puede realizarse bajo condiciones de con y sin conversión de longitud de onda (RCC $\lambda$ Y RSC $\lambda$ ). Las redes ópticas pueden tener la capacidad de asignar la misma o diferente longitud de onda para las rutas, de esta forma existen redes que asignan diferentes longitudes de onda o también llamadas con reutilización de longitud de onda (RCC $\lambda$ ) y redes que asignan la misma longitud de onda (RSC $\lambda$ ) (Balasis et al., 2013; Rodríguez et al., 2010). Las diferencias entre estas configuraciones, radican básicamente en una mayor disponibilidad de establecimiento de la conexión en las RCC $\lambda$, determinando una disminución del indicador utilización de la red, sin embargo el cambio de longitud de onda trae un mayor nivel de retardo. Las RSC $\lambda$ establecen una restricción conocida en la literatura como CCW (Continuity Constraint Wavelength); esta restricción ayuda a mejorar el retardo en la ruta de la demanda presente (Rodríguez et al., 2011). Sin embargo el indicador utilización de la red aumenta, disminuyendo la satisfacción de la demanda futura; ya que los caminos se agotan mientras más rutas se establezcan.

El problema RWA se ha resuelto desde dos estrategias, con solución dividida (ESD) o con solución integral (ESI). La ESD divide el problema en dos partes donde primero se obtiene la ruta y segundo se asigna la longitud de onda y la ESI soluciona el problema en un solo proceso algorítmico. Para la obtención de la ruta se utilizan algoritmos heurísticos (Tráfico dinámico) tales como: Simulated Annealing (SA), Algoritmos Genéticos (AG) y Taboo Search (TS) (Barpanda et al., 2011; Rodríguez et al., 2011; Rodriguez et al., 2014). Para la asignación de longitud de onda se utilizan algoritmos muy conocidos de la asignación de memoria en los sistemas operativos de computadores tales como: First Fit (FF), More Fit (MF), Random Fit (RF) y Next FIT (NF) (Barpanda et al., 2010; Rodríguez et al., 2011). El presente artículo muestra los resultados de una nueva heurística comparada con los tres trabajos anteriormente mencionados. Este procedimiento es denominado Algoritmo Snake que se describe más adelante. Los resultados muestran grandes mejoras en el indicador probabilidad de bloqueo, pero con un aumento de la utilización de la red.

\section{RWA - ROUTING WAVELENGTH ASSIGNMENT}

Las redes WDM están formadas por conmutadores ópticos, denominados OXC (Optical Cross Connect) o nodos, enlazados por fibras ópticas, que tienen características que permiten diferentes longitudes transitando simultáneamente, es decir fibras ópticas de baja dispersión para controlar los fenómenos no lineales. Dependiendo de la tecnología de estos conmutadores pueden conmutar fibras, longitudes de onda, bandas de longitudes de onda, canales de tiempo TDM [(Fukuda et al., 2014; Gu et al., 2015)].

Las rutas están formadas por enlaces $(e)$ adyacentes que dependerá del origen y destino de la solicitud. En la ecuación 1 , se puede observar la ruta $0(R o)$ cuyo origen y destino es el nodo 4 y 9 respectivamente, formada por cuatro enlaces.

$R_{0}=\left(\mathrm{e}_{41}, \mathrm{e}_{15}, \mathrm{e}_{57}, \mathrm{e}_{79}\right)$

Los $L P$ se forman al asociar una ruta $(R)$ con una longitud de Onda $(\lambda)$, esto se puede observar en la ecuación 2.

$L P=(R, \lambda)$ 
En las redes RSC $\lambda$ se debe establecer el CCW, que determina la misma longitud de onda en cada enlace a lo largo de la ruta, sin embargo a medida que se establecen los LP la posibilidad de establecer uno nuevo se va agotando y cuando no se satisface la CCW la solicitud es bloqueada. Existen estudios para redes RCC $\lambda$ llamados con reutilización de la longitud de onda, pero las investigaciones muestran cambios poco significativos en el desempeño del enrutamiento (Hredzak et al., 2011).

En la Figura 1, se observan siete conmutadores ópticos, donde se han establecido dos LP; el primero ( $e_{67}-e_{74}$ $\left.-e_{41},-\lambda_{0}\right)$ que utiliza $\lambda_{0}$ y el segundo $\left(e_{36}-e_{67}-e_{75}-e_{52},-\lambda_{1}\right)$ que utiliza $\lambda_{1}$. Se puede ver que el enlace $e_{67} e$ común a los dos y el sistema solo tienen dos longitudes de onda, por lo tanto ese enlace se encuentra saturado. Si una solicitud es satisfecha con un LP que contenga dicho enlace determina la aparición del fenómeno de contención por lo que se deberá utilizar otra longitud de onda o en su defecto buscar una nueva ruta.

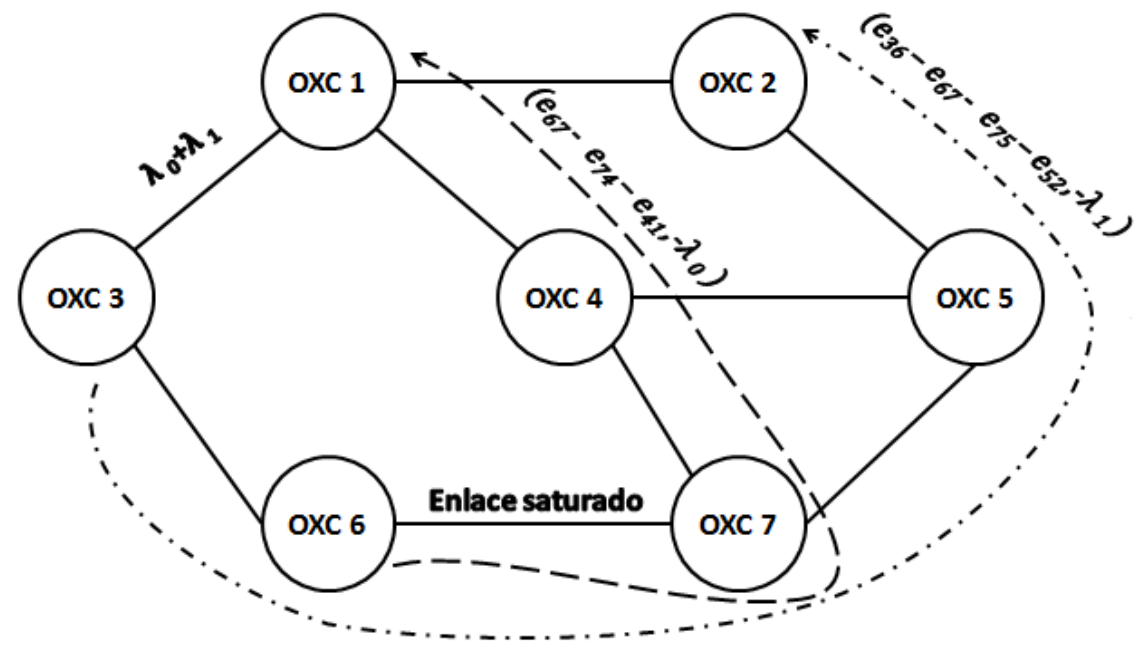

Fig. 1: LightPath en redes ópticas con dos longitudes de onda.

Para efectos descriptivos, se establece que existe un tráfico actual (CT- Current Traffic), un tráfico entrante (IT - Incoming Traffic) una demanda futura, dependiendo de las características de los tiempos de conexión solicitados (HT - Holding Time) y el tiempo entre llegadas de solicitudes, se determinará si el tráfico es estático o dinámico (Rodríguez et al., 2014). Para la solución del RWA, se han ensayado diferentes estrategias (dividida e integral) en escenarios estáticos y dinámicos; diferentes criterios de optimización tales como la minimización de la probabilidad de bloqueo de nuevas solicitudes, minimización de la utilización de las longitudes de onda de la red, minimización del uso de longitudes de onda por enlace, minimización de la dispersión en la ruta, minimización del ruido ASE (Amplified Spontaneous Emission) en la ruta entre otros (Monoyoios et al., 2011; Charbonneau et al., 2010). Los algoritmos de optimización han sido clasificados en convencionales y heurísticos. Los algoritmos heurísticos más utilizados son: Algoritmo Hormiga (Tan et al., 2012; Zhang-liang et al., 2011), Genético (Castrillón et al., 2011; Rashedi et al., 2012; Rodriguez et al., 2014), Búsqueda Tabú (Boonyopakom et al., 2011; Khelifi et al., 2013; Rodríguez et al., 2014) y Simulated Annealing (Sakamoto et al., 2013; Na et al., 2011; Rodríguez et al., 2010). Mayor información sobre las estrategia, criterios y algoritmos heurísticos, puede ser revisada en (Rodríguez et al., 2014]. Se debe resaltar, que en las redes ópticas WDM no se busca optimizar (tráfico dinámico), solo e requiere ruta que funcione, sin generar un aumento importante de la probabilidad de bloqueo y sin aumento de la utilización de la red.

\section{DESCRIPCION DEL ALGORITMO}

El algoritmo propuesto ha sido denominado Snake-One, y fue comparado con el algoritmo Simulated Annealing que utiliza una estrategia basada en el principio de enfriamiento de los materiales, en su paso a lograr estados de cristalización; el algoritmo genético que utiliza una estrategia basada en el proceso de evolución biológica basado en su base genética, es una serie de pasos que realizan reproducción, ordenamiento, mutación y mortandad y el algoritmo Búsqueda Tabú que utiliza una estrategia de premio y castigo a los cambios del proceso en la matriz de costos (Rodríguez et al., 2014).

El nuevo proceso heurístico simula el movimiento de una serpiente dentro de una matriz que representa el estado de los costos de la red óptica. La ruta se construye con movimientos horizontales y verticales en la matriz denominada Snake, en cada cambio de dirección se construye la ruta hasta encontrar el nodo destino, luego se utiliza el algoritmo FF para la asignación de longitud de onda, conformando de esta manera el LP buscado. 
En toda red óptica los nodos de borde son conocidos como Edge OXC, y son los que reciben el IT (con distribución Poisson) dicho tráfico está caracterizado con cuatro parámetros que deben ser satisfechos, de lo contrario la solicitud deberá ser bloqueada, nodo origen, nodo destino, número de conexiones y el HT. Se define $\boldsymbol{N}$ como el número de nodos activos en la red óptica, $\boldsymbol{n}_{C X}$ es el número de conexiones máximo en cada enlace, $\boldsymbol{n}_{W}$ es el número de Longitudes de Onda en cada enlace (supuesto iguales en todos los enlaces de la red).

Se generan cinco matrices, la matriz de enlaces $E$, la matriz de costos $C$, la matriz de Lambdas $\lambda$, la matriz de tiempos $T$ y la matriz $S$ (Snake). Fueron adicionadas dos columnas en la matriz $S$ al inicio y al final, con la finalidad de guardar cálculos parciales como costos totales de las filas y la etiqueta del nodo, estos modelos pueden ser revisados con profundidad en (Rodríguez et al., 2014), los mismos que fueron utilizados como comparación.

$$
S=\left\{\begin{array}{cl}
s_{i 0 k}=i & \forall i \in[0, N-1] \wedge k \in\left[0, n_{w}-1\right] \\
s_{i j k} / s_{i j k}=c_{i j k} * t_{i j k} & \forall i \in[0, N-1] \wedge j \in[1, N] \wedge k \in\left[0, n_{w}-1\right] \\
s_{i(N+1) k}=\sum_{j=1}^{N} s_{i j k} & \forall i \in[0, N-1] \wedge j \in[1, N] \wedge k \in\left[0, n_{w}-1\right]
\end{array}\right\}
$$

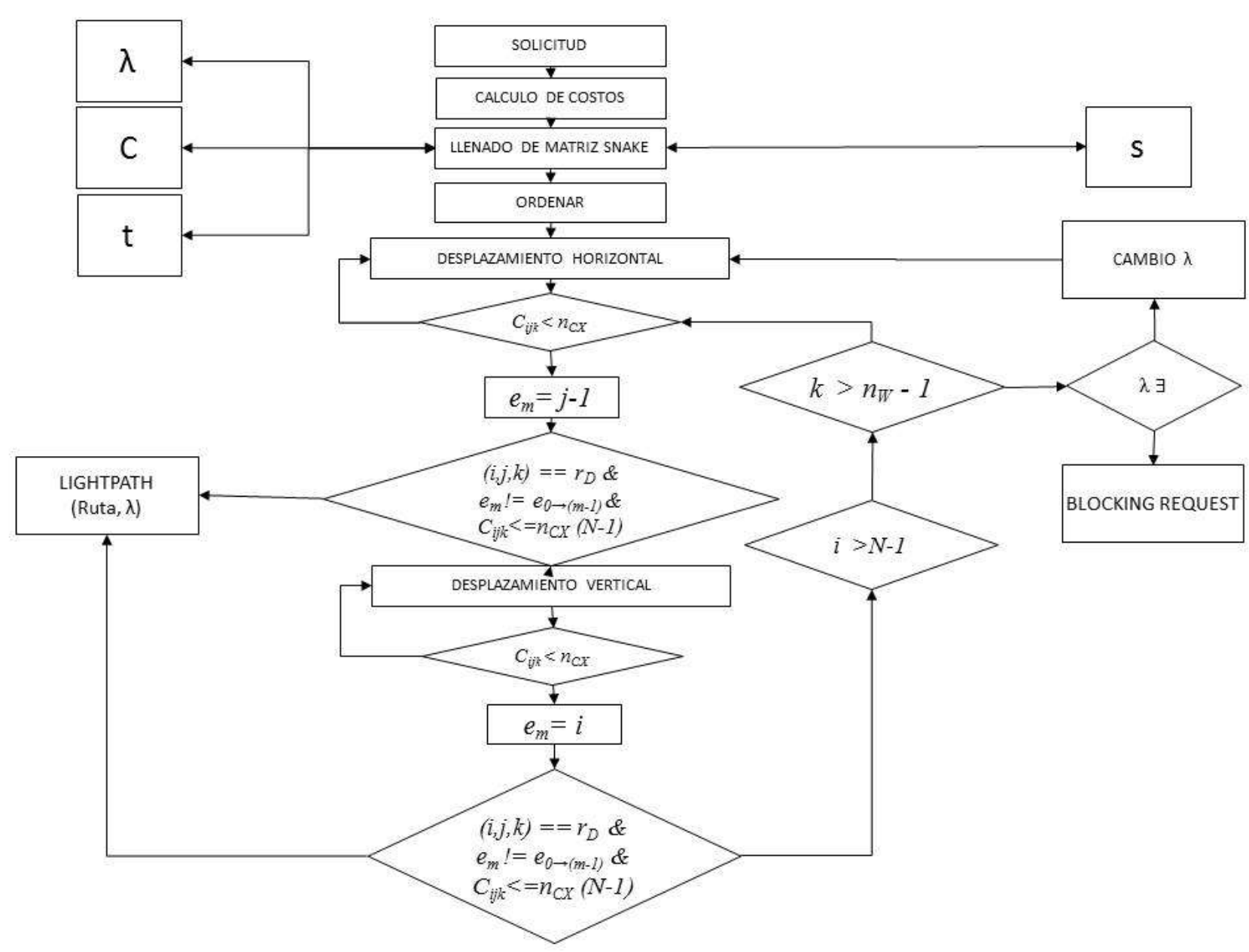

Fig. 2: Diagrama de flujo del algoritmo Snake

En la Figura 2, podemos observar los procedimientos para obtener el LP y en la Figura 3, se observa un ejemplo de la matriz Snake para una red de ocho nodos. Se observa que en la primera columna se encuentran los nodos de la red y en la última columna los costos totales por fila, ambas columnas fueron adicionadas para tal efecto. 


$$
S_{i j}=\begin{array}{cccccccccc}
\text { Node } & \mathbf{0} & \mathbf{1} & \mathbf{2} & \mathbf{3} & \mathbf{4} & \mathbf{5} & \mathbf{6} & \mathbf{7} & \text { Cost } \\
\mathbf{0} & 1 & 2 & 3 & 4 & 5 & 6 & 7 & 8 & 9 \\
0 & 0 & 16 & 16 & 1000 & 1000 & 1000 & 16 & 16 & 3064 \\
1 & 16 & 0 & 16 & 16 & 16 & 1000 & 1000 & 1000 & 3064 \\
4 & 1000 & 16 & 1000 & 16 & 0 & 16 & 16 & 1000 & 3064 \\
6 & 16 & 1000 & 1000 & 1000 & 16 & 16 & 0 & 16 & 3064 \\
2 & 16 & 16 & 0 & 1000 & 1000 & 16 & 1000 & 1000 & 4048 \\
3 & 1000 & 16 & 1000 & 0 & 16 & 1000 & 1000 & 16 & 4048 \\
5 & 1000 & 1000 & 16 & 1000 & 16 & 0 & 16 & 1000 & 4048 \\
7 & 16 & 1000 & 1000 & 16 & 1000 & 1000 & 16 & 0 & 4048
\end{array} \mid
$$

Fig. 3: Matriz Snake de la longitud de onda 0.

En la matriz S se aplicará el algoritmo Snake y se buscará una ruta si no se encontrase se procederá a buscar ruta en la longitud de onda siguiente (FF), si no se encuentra ruta en las longitudes de onda disponibles se procede a bloquear la solicitud. La disponibilidad temporal de la longitud de onda es administrada en la matriz $\mathrm{T}$ y sus elementos son valorados con 1 cuando hay disponibilidad. Sea la solicitud $d_{12}^{2}=(2,5,2,1200)$, que representa a la doceava solicitud que llega al nodo 2 con destino al nodo 5 , solicitando 2 conexiones durante 1200 ms. A continuación se ordena descendentemente la Matriz S respecto de la columna de costos. Observando la columna 0 de la matriz $S$ (Nodos) se ubica en la fila correspondiente al Nodo Origen $2(4,0)$, como se puede observar en la Figura 6. Luego se procede al desplazamiento horizontal hasta ubicar el primer costo mayor a $n_{c}$ y menor a 1000, (Valor que permite diferencia enlace no existente) ubicándose en el nodo 0 $(4,1)$. Como el nodo 0 no corresponde al destino, se procede al desplazamiento vertical hasta ubicar un costo mayor a 0 y menor a 1000 ubicándose en el nodo 7 (7,1). Como el nodo 7 no corresponde al destino, se procede al desplazamiento horizontal nuevamente, ubicando el Nodo $3(7,4)$, luego desplazamiento vertical ubicándonos en el nodo $4(2,4)$ y luego se procede al desplazamiento horizontal ubicando el Nodo destino 5 $(2,6)$ dando termino al algoritmo (Figura 4), por ser el destino propuesto en la solicitud entrante.

$$
S_{i j}=\begin{array}{cccccccccc}
\text { Node } & \mathbf{0} & \mathbf{1} & \mathbf{2} & \mathbf{3} & \mathbf{4} & \mathbf{5} & \mathbf{6} & 7 & \text { Cost } \\
\mathbf{0} & 1 & 2 & 3 & 4 & 5 & 6 & 7 & 8 & 9 \\
0 & 0 & 16 & 16 & 1000 & 1000 & 1000 & 16 & 16 & 3064 \\
1 & 16 & 0 & 16 & 16 & 16 & 1000 & 1000 & 1000 & 3064 \\
4 & 1000 & 16 & 1000 & 16 & 0 & 16 & 16 & 1000 & 3064 \\
6 & 16 & 1000 & 1000 & 1000 & 16 & 16 & 0 & 16 & 3064 \\
2 & 16 & 16 & 0 & 10 p 0 & 1000 & 16 & 1000 & 1000 & 4048 \\
3 & 1000 & 16 & 1000 & \oint_{0} & 16 & 1000 & 1000 & 16 & 4048 \\
5 & 1000 & 1000 & 16 & 10 p 0 & 16 & 0 & 16 & 1000 & 4048 \\
7 & 16 & 1000 & 1000 & 16 & 1000 & 1000 & 16 & 0 & 4048
\end{array} \mid
$$

Fig. 4: Desplazamiento Vertical para la longitud de onda 0

Por lo tanto el desplazamiento en la matriz $S$ es $(4,0) \rightarrow(4,1) \rightarrow(7,1) \rightarrow(7,4) \rightarrow(2,4)) \rightarrow(2,6)$. Esto corresponde al lightpath 2-0-7-3-4-5, con la longitud de onda 0 , como se observa en la Figura 5.

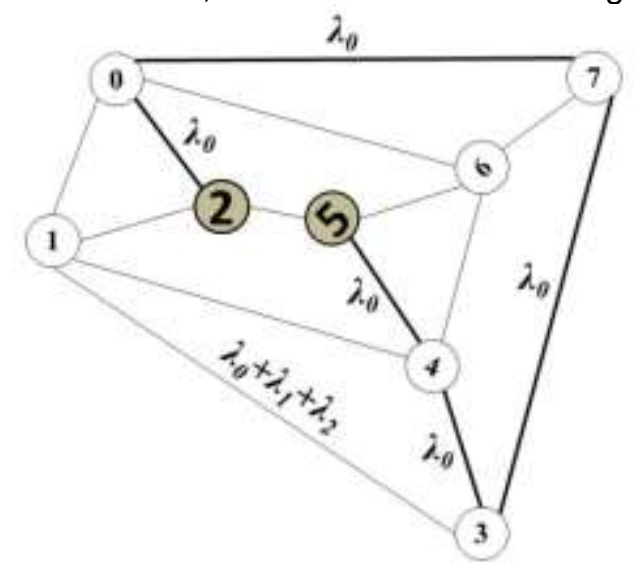

Fig. 5: Solución para la red ejemplo de 3 longitudes de onda $\lambda_{0}, \lambda_{1}, \lambda_{2}$. 
Al observar la conducta del algoritmo se ve que obtiene la ruta recorriendo y consumiendo muchos recursos de la red. Es decir, obtener la ruta con mucha rapidez es a costo de utilizar muchos recursos de la red.

\section{ANALISIS DE LOS RESULTADOS}

Los resultados de la simulación fueron comparados con trabajos de en igualdad de condiciones en la red NSFNET, de 16 nodos y 25 enlaces de fibra óptica. Las comparaciones fueron realizadas utilizando los indicadores de probabilidad de bloqueo y utilización de la red. La carga fue incrementada a razón de 10 Erlangs por simulación, llegando hasta los 180 Erlangs. La cantidad de conexiones realizadas por simulación en los algoritmos Simulated Annealing (SA), Genéticos (AG), Búsqueda Tabú (AG) y Snake-One (SNK1) fue de $10^{8}$ solicitudes de conexión, lo que permitió someter a stress los algoritmos.

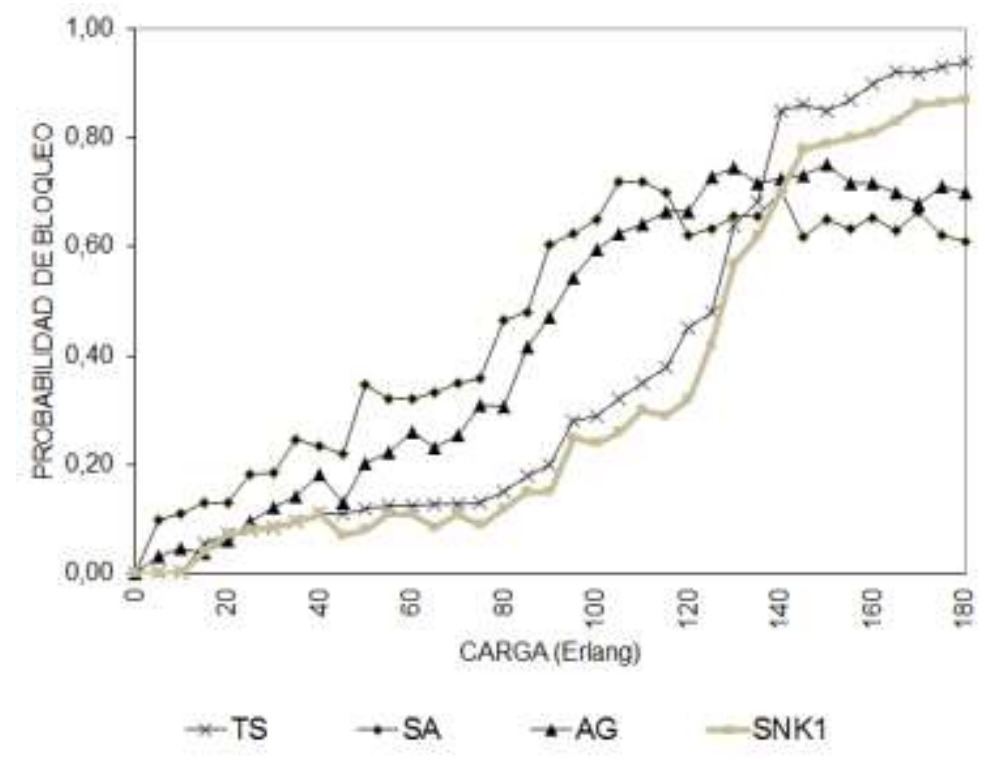

Fig. 6: Comparación de la Probabilidad de Bloqueo en la NSFNET.

En la Figura 6, se muestra la comparación de los algoritmos heurísticos respecto del indicador probabilidad de bloqueo, notándose la mejora del indicador hasta los 140 Erlang, superando al resto de heurísticas. Este comportamiento ayuda a la red a satisfacer una solicitud de servicio de manera rápida y sobre todo oportuna, a pesar del alto consumo de recursos de la red.

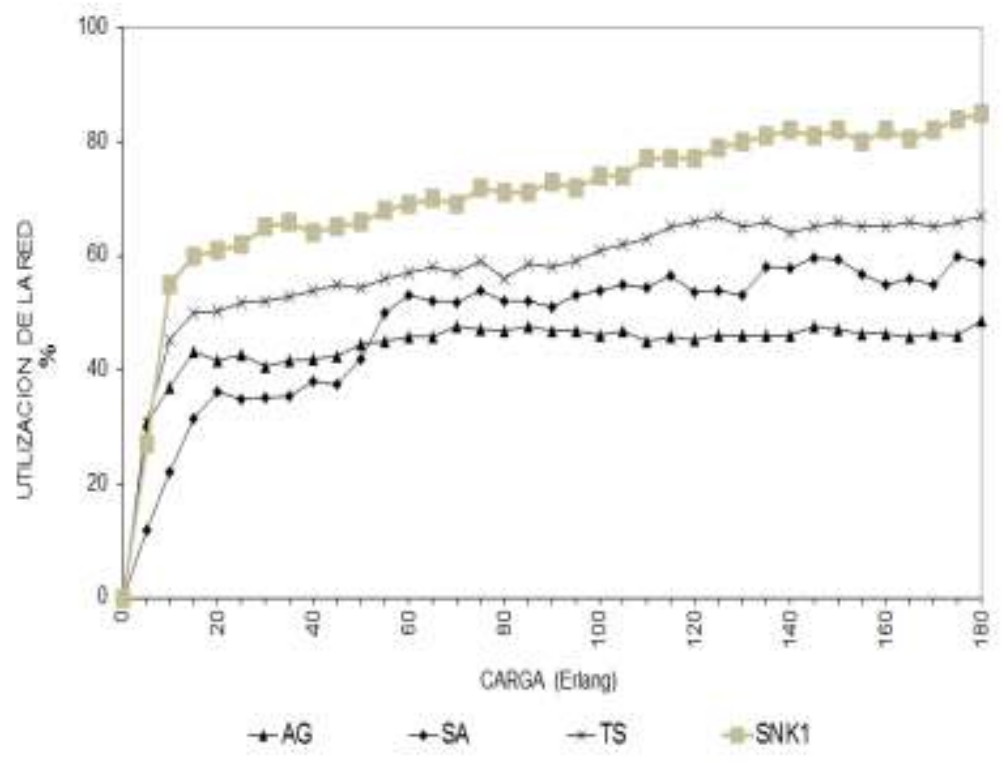

Fig. 7: Comparación de la utilización de red en la NSFNET. 
En la Figura 7, se muestra la comparación de los algoritmos heurísticos respecto del indicador utilización de la red, que hace visible el gran consumo de recursos del algoritmo, frente al resto de heurísticas. En estas condiciones, la única forma de mejorar el algoritmo es el aumento de longitudes de onda, esto permitirá una mejor respuesta a la demanda futura, con la consecuencia de un aumento en los costos del servicio.

\section{CONCLUSIONES}

Es notoria la mejora del indicador probabilidad de bloqueo aún a pesar de un alto consumo del indicador utilización de la red, esto determina satisfacción al "incomming traffic" a costa del "future traffic", esto no resulta tan severo debido ya que en las redes ópticas las preocupaciones apuntan a atender las solicitudes entrantes con eficiencia y oportunidad, debido a que las posibilidades que aparezca "burst traffic" (tráfico ráfaga no uniforme) son menores y poco frecuentes. Frente a este hecho se establecen dos soluciones que serán materia de estudio más adelante, la algoritmia Snake-One abre un camino hacia la solución del problema RWA y se vislumbran dos modificaciones (Snake-Two y Snake-Three), ambas procurarán mejorar el indicador de utilización de la red, con poco aumento de la probabilidad de bloqueo, estas modificaciones incorporarán al proceso una matriz de costo instantáneo de enlaces (Snake-Two) y una matriz de costo instantáneo de nodos (Snake-Three), que procurará dirigir el uso de la red de forma ordenada, de esta forma se mantendrán saturados algunos enlaces pero otros se liberarán, logrando aumentar la disponibilidad para atender la demanda futura, con el consecuente aumento del indicador utilización de la red.

\section{AGRADECIMIENTOS}

Para el Programa DYCYT de la Universidad de Santiago de Chile- USACH (Project DICYT N 061572RG), por su importante apoyo al desarrollo de la investigación y al Grupo de Investigación en Nuevas Tecnologías (GINT-DTI-USACH).

\section{REFERENCIAS}

Assis K. D. R., Ferreira dos Santos A. y Giozza W. F., Hybrid Algorithms for Routing and Assignment Wavelengths in Optical Networks. Latin America Transactions, 8(1), 214-220 (2010)

Balasis F., Wang X., Xu S. y Tanaka Y., A dynamic physical impairment-aware routing and wavelength assignment scheme for 10/40/100 Gbps mixed line rate wavelength switched optical networks, Advanced Communication Technology (ICACT), 2013 15th International Conference on, 116- 121 (2013)

Barpanda R. S., Sahoo B., Turuk A. K. y Majhi B., Genetic Algorithm techniques to solve Routing and Wavelength Assignment problem in Wavelength Division Multiplexing all-optical networks. Communication Systems and Networks (COMSNETS). 1, 1-8 (2011)

Barpanda R. S., Sahoo B., Turuk A. K. y Majhi B., Solving large problem instances of the RWA problem using Genetic Algorithms. Industrial and Information Systems (ICIIS). 1, 41-46 (2010)

Boonyopakorn P. y Meesad P.,A Hybrid GA and Tabu Search Approach to Find Optimal Node Placement in IP Networks. Wireless Communications, Networking and Mobile Computing (WiCOM). 1, 1-4 (2011)

Castrillón O., Sarache W. y Giraldo J., Parámetros Numéricos en el Método de los Algoritmos Genéticos para el Sublimación. Inf. tecnol. [online], 22(1), 83-92 (2011)

Charbonneau N. y Vokkarane V. M., Static Routing and Wavelength Assignment for Multicast Advance Reservation in All-Optical Wavelength-Routed WDM Networks. Networking. IEEE/ACM Transactions on. Vol. 20, pp. 1-14 (2012)

Charbonneau N. y Vokkarane V. M., Tabu Search Meta-Heuristic for Static Manycast Routing and Wavelength Assignment over Wavelength-Routed Optical WDM Networks. Communications (ICC). 1, 1-5 (2010)

Fukuda T., Liu, L., Baba, K., Shimojo, S. y Yoo S., GMPLS Control Plane with Distributed Multi-path RMSA for Elastic Optical Networks. Journal of Lightwave Technology. 99, 1-9 (2015)

Gu X., Susuki K., Ikuma Y., Seno K., Tanobe H., Matsutani A., y Koyama F., Compact Wavelength Selective Switch Using a Bragg Reflector Waveguide Array with Ultra-Large Number $(>100)$ of Output-Ports. Journal of Lightwave Technology. 99, 1-7 (2014) 
Hredzak B. y Diessel O., Optimization of placement of dynamic network-on-chip cores using simulated annealing. IECON 2011 IEEE. 1,. 2400-2405 ( 2011)

Khelifi L., Zidi I., Zidi K. y Ghedira K., A hybrid approach based on Multi-Objective Simulated Annealing and Tabu Search to solve the Dynamic dial a Ride Problem. Advanced Logistics and Transport (ICALT). 1, 227$232(2013)$

Monoyios D. y Vlachos K., Multiobjective Genetic Algorithms for Solving the Impairment-Aware Routing and Wavelength Assignment Problem. Optical Communications and Networking. 3, 40-47 (2011)

Na L., Haixing L. y Luo Y., A New MPLS Fault Restoration Algorithm Based on Simulated Annealing and Tabu Search. Wireless Communications, Networking and Mobile Computing (WiCOM). 1, 1-4 (2011)

Rashedi Y., Kavia S., Mahani A. y Strobel O., Evolutionary algorithms for solving routing and wavelength assignment problem in optical networks: A comparative study. Transparent Optical Networks (ICTON). 1, 1-4 (2012)

Rodriguez A., Gutierrez A., Rivera L. y Ramirez L., Ruteo y Asignación de Longitud de Onda: Comparación de Algoritmos Genéticos y Simulated Annealing. Inf. tecnol. 4, 13-18 (2014)

Rodriguez A., Ramirez L., Rivera L. y Gutierrez A., Routing wavelength assignement: A solution with tabu search in dynamic traffic. Ingeniare. Rev. chil. ing. 4, 495-503 (2014)

Rodriguez, A. y Saavedra F., Optimización del Algoritmo Genético para la Solución Integral de Enrutamiento en Redes Fotónicas. Inf. tecnol. [online], 21(3), 125-133 (2010)

Sakamoto S., Oda T., Kulla E., Ikeda M., Barolli L. y Xhafa F., Performance Analysis of WMNs Using Simulated Annealing Algorithm for Different Temperature Values. Complex, Intelligent, and Software Intensive Systems (CISIS). 1, 164-168 (2013)

Tan S., Ant-based Physical Attack and Amplifier Spontaneous Emission-aware routing, Communication Technology (ICCT), 2012 IEEE 14th International Conference on, 650- 653 (2012)

Zhang-liang W. y Yue-guang L., An Ant Colony Algorithm with Tabu Search and its Application. Intelligent Computation Technology and Automation (ICICTA). 1, 412-416 (2011) 